\title{
METODOLOGIA DE ANÁLISE E DIAGNÓSTICO DA MADEIRA NA PRESERVAÇÃO DO PATRIMÔNIO HISTÓRICO
}

\author{
Janice Bernardo da Silva ${ }^{1}$; Márcio Pereira da Rocha ${ }^{2}$; Key Imaguire $\mathrm{Jr}^{3}$ \\ ${ }^{1}$ Arquiteta e Urbanista, M.Sc., Doutoranda em Eng. Florestal, UFPR, Curitiba, PR, Brasil - janicebs@ gmail.com \\ ${ }^{2}$ Eng. Florestal, Dr., Depto. de Engenharia e Tecnologia Florestal, UFPR, Curitiba, PR, Brasil - mprocha@ufpr.br \\ ${ }^{3}$ Arquiteto e Urbanista, Dr., Depto. de Arquitetura e Urbanismo, UFPR, Curitiba, PR, Brasil - keyimajun@ufpr.br
}

Recebido para publicação: 16/02/2009 - Aceito para publicação: 01/10/2009

\begin{abstract}
Resumo
O panorama atual das edificações históricas de madeira é marcado pelas degradações intensas associadas ao clima tropical brasileiro, além da falta de consciência cultural de preservação. Projetos de restauração contemplam diagnósticos eficazes que levam à suspensão das causas geradoras da deterioração, de modo a garantir a reabilitação e a integridade das obras às futuras gerações. Para a consecução deste trabalho, optou-se pelo exame da casa Grochocki, localizada na colônia Murici, Região Metropolitana de Curitiba, construída de troncos de Araucaria angustifolia, remanescente da tradição construtiva polonesa e da arquitetura de madeira do Paraná. Foi intuito deste estudo formular uma metodologia de análise e uma ficha de avaliação do estado de conservação da edificação em questão. Foram realizados análises visuais, o mapeamento das degradações e a análise não-destrutiva com o equipamento Stress wave timer. Uma classe de deterioração foi gerada a partir dos dados qualitativos do equipamento, e diferentes profissionais testaram a aplicabilidade da ficha. Obtiveram-se resultados positivos para avaliação da madeira no patrimônio histórico. Pôde-se verificar também que os mais aptos para este diagnóstico demonstraram ser os profissionais que tiveram seus conhecimentos aprofundados em aspectos tecnológicos e patológicos da madeira.

Palavras-chave: Patrimônio; madeira; metodologia de análise.
\end{abstract}

\begin{abstract}
Methodology analysis and diagnosis of wood in preservation historical patrimony. The actual scenery of historical wood buildings is characterized by intense degradation related to the tropical brazilian weather, besides the absence of cultural preservation. Projects of restorations need efficient diagnosis to present good results in stopping the causes of degeneration, and in preserving the architeturals works to the new generations. This research analyses the house called «Grochocki» situated at the Murici Colony, near Curitiba, built by roof timber, trunk and board, with the brazilian wood called Araucaria angustifolia. The «logs home» is a remanent of the traditional polish colonization and the local architectural wood construction of Paraná. This study presents a methodology of analysis and a valuation card considering the preservation. It considers also a visual analysis, a degradation mapping and a non-destructive analysis with the Stress wave timer equipment. One category of deterioration was created and different professionals testified the relevance of the card. Good results in evaluation of the wooden historical patrimony were found. The best prepared professionals capable to realize the diagnosis, proved to be those with the largest kwnoledges in technological and pathological aspects of wood.

Keywords: Architectural patrimony; wood; methodology analysis.
\end{abstract}

\section{INTRODUÇÃO}

A conservação do patrimônio em madeira no Brasil sofre pela ação da degradação dos agentes externos associados ao clima tropical. Há falta de conhecimento e de tradição do tema "restauração" e da consciência cultural de preservação. As casas de troncos construídas pelo imigrante polonês no sul do Brasil no século XIX constituem marco histórico na produção arquitetônica, principalmente no Paraná. As casas de troncos encontradas na Colônia Murici, no município de São José dos Pinhais, constituem aspectos culturais e conhecimentos da tradição das antigas construções de madeira da Europa setentrional, em especial da Polônia. 
Regionalmente, a madeira utilizada foi a Araucaria angustifolia, classificada como uma espécie não durável ou de baixa resistência (suscetível aos organismos deterioradores). A madeira está sujeita a deteriorações rápidas, pela ação do fogo, ou mais lentas, pela ação dos agentes atmosféricos ou biológicos (LIOTTA, 1998). De natureza abiótica, as alterações físicas são causadas por intempéries, a radiação solar, os poluentes e a umidade. De natureza biótica, as alterações correspondem ao ataque por fungos e insetos. A degradação biológica provoca danos tanto às obras recentes como às antigas, trazendo grande prejuízo ao acervo mundial.

Segundo a Carta de Restauro de 1972, o projeto de restauração de uma obra arquitetônica deverá ser precedido de um exaustivo estudo sobre o monumento (IPHAN, 1995). Para obras de madeira, Tampone (1996) sugere a conservação, reservando ao restauro o caráter de intervenção excepcional, no espírito das Cartas Patrimoniais e a partir de orientações atualizadas. Como procedimento metodológico, recomenda o conhecimento das características químicas e físicas, em particular as mecânicas e as deformações do material em estruturas, a ciência dessas tipologias e do seu respectivo comportamento.

Os métodos não-destrutivos são os preferencialmente empregados no setor de bens culturais. Justifica-se, no entanto, segundo Arosio (2003), a utilização de métodos destrutivos, ou preferencialmente os microdestrutivos, tendo em vista a elaboração de técnicas capazes de bloquear os processos de degradação, que levariam a danos irreversíveis. O levantamento que precede a uma análise deve ser detalhado para a escolha de uma amostra, sendo em ponto significativo e que forneça conteúdo expressivo.

O Osservatorio per la Conservazione delle Opere Lignee ${ }^{1}$, que desenvolve atividades de diagnóstico de obras históricas, entre outras utiliza a metodologia descrita no manual "La diagnosi delle opere e delle strutture lignee: Le Inspezioni" (AUGELLI, 2006). O manual se embasa em normas técnicas da madeira e de restauração de bens culturais e segue as normas da $\mathrm{UNI}^{2}$ (Ente Nazionale Italiano di Unificazione), as recomendações do ICOMOS (International Council on Monuments and Sites $^{3}$ ) e outras normas europeias.

Propõe-se, nesta investigação, a formulação de uma metodologia de análise e diagnóstico que vise à preservação dos bens imóveis de madeira por meio de técnicas de análise não-destrutivas, além da aplicação de ficha de avaliação e teste de verificação dos profissionais mais aptos ao diagnóstico.

\section{MATERIAL E MÉTODOS}

Para a consecução do presente estudo, optou-se pelo exame de uma casa de troncos, sendo avaliados elementos arquitetônicos de diferentes seções, como troncos, tábuas, caibros, vigas, toretes, tanto elementos de vedação quanto estruturais.

Para a formulação da metodologia proposta, partiu-se das análises do objeto (arquitetura) e da matéria (madeira), com levantamentos realizados no local. Através de análise visual, foram examinadas as degradações e os defeitos, e o estado de conservação da edificação ficou então registrado de forma gráfica, no mapeamento.

Obtiveram-se dados da integridade das peças por análise não-destrutiva. O equipamento usado foi o de emissão de ondas de tensão (stress wave timer), do Laboratório de Tecnologia da Madeira e Produtos da Madeira da UFPR (Figura 1).

Segundo Emerson et al. (1998, apud GORNIAK; MATOS, 2002), a aplicação e medição por ondas de tensão consiste na colocação de dois transdutores nas extremidades de uma peça em sentido longitudinal. A emissão de onda por meio de martelo ou pêndulo entre o acelerômetro de partida e chegada fornecerá uma contagem de tempo em microssegundos, através da peça. A variação do tempo percorrido pela onda revelará as condições internas do material. Havendo coesão das células, ou seja, em material sadio, o tempo é menor, enquanto que na madeira deteriorada o tempo é maior. Quão intensamente presentes estiverem as descontinuidades, os nós, as resinas e as deteriorações, maiores serão os valores lidos.

1 Laboratório de Conservação de Obras de Madeira, docente responsável Francesco Augelli, Facoltà di Architettura Civile Politécnico de Milão (N. A.).

2 A Itália foi o primeiro país, não só na Europa, mas em todo o mundo, a adotar uma normativa específica para a conservação dos bens que constituem o patrimônio inalienável de cada nação (AUGELLI, 2006, p. 19) - (T. A.).

3 International Council on Monuments and Sites - Conselho Internacional de Monumentos e Sítios, órgão responsável pela preservação do patrimônio mundial ligado à UNESCO (N.A.). 


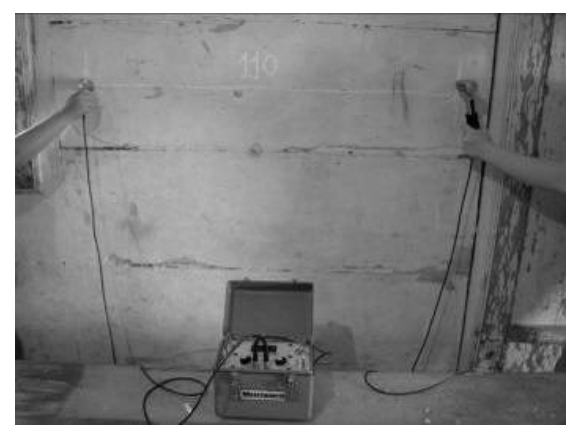

Figura 1. Utilização do equipamento stress wave timer.

Figure 1. Use of stress wave timer equipment.

Os valores de tempo de propagação de todas as peças foram transformados em velocidade (Tabela 1), e sua comparação gerou uma nota de classificação de 0 a 3 . Ao valor mínimo foi designada a classificação (3) - severamente deteriorada - e ao valor máximo foi designada a classificação (0) - não deteriorada - em uma faixa numérica de 750 unidades. Para os valores intermediários, a classificação (2) - deteriorada - e (1) levemente deteriorada. A divisão numérica foi aleatória, mas considerou alguns parâmetros da Norma UNI EN 335, citada por Augelli (2006), a qual contempla a definição das classes de risco biológico, dada a probabilidade de degradação por organismos xilófagos em relação às condições ambientais em que a madeira se encontra.

Tabela 1. Cálculo da velocidade e a classificação por classes de deterioração.

Table 1. Calculation of speed and classification by classes of deterioration.

\begin{tabular}{lcccccc}
\hline Elemento & & $\begin{array}{c}\text { Distância } \\
(\mathbf{d}) \mathbf{m}\end{array}$ & $\begin{array}{c}\text { tempo (t) } \\
\text { microsseg. }\end{array}$ & $\begin{array}{c}\text { Tempo (t) } \\
\text { segundos }\end{array}$ & $\begin{array}{c}\text { Velocidade (v) } \\
(\mathbf{d} / \mathbf{t}) \mathbf{~ m} / \mathbf{s}\end{array}$ & Classificação \\
\hline Base & B2 & 0,30 & 1923,0 & 0,001923 & 156 & 3 \\
& B4 & 0,35 & 496,3 & 0,000496 & 705 & 3 \\
Parede & B1 & 1,60 & 7385,0 & 0,007385 & 217 & 3 \\
Frontal externa & B4 & 1,60 & 1605,7 & 0,001605 & 996 & 2 \\
Piso térreo & A14 & 4,50 & 946,0 & 0,007946 & 566 & 3 \\
& A20 & 4,50 & 1700,3 & 0,001700 & 2647 & 0 \\
\hline
\end{tabular}

Essa classe de valores subsidiou a ficha de avaliação (Figura 2), que constituiu elemento de classificação de operadores aptos à realização de diagnósticos de edificações históricas. A partir de 27 elementos de madeira selecionados, 18 indivíduos estiveram no local e utilizaram a ficha, analisando a edificação. Posteriormente os valores fornecidos foram comparados com o grupo testemunha. Entre os avaliadores estavam arquitetos, engenheiros, restauradores, designers, biólogos, estudantes e leigos, organizados em 6 grupos, conforme a sua formação, experiência profissional e conhecimentos na área da madeira e de restauração.

Segue a descrição dos grupos:

Grupo-testemunha: autora e o stress wave timer.

Grupo A: Arquitetos, engenheiros, biólogos, com especialização em restauração e estudos na área de deterioração da madeira.

Grupo B: Arquitetos, engenheiros, designers, pós-graduados em engenharia florestal - área de tecnologia e utilização de produtos florestais, sem especialização na área de restauração.

Grupo C: Arquitetos com experiência e/ou especialização em restauração, sem estudos na área de tecnologia e deterioração da madeira.

Grupo D: Arquitetos, sem especialização ou experiência em restauração e sem estudos na área de tecnologia e deterioração da madeira.

Grupo E: Estudantes do curso de biologia, engenharia florestal e engenharia industrial madeireira, sem especialização na área de restauração. 
Grupo F: Avaliadores sem formação ou experiência em qualquer das áreas supracitadas.

A natureza classificatória da metodologia aplicada fez com que se utilizasse o teste $\mathrm{H}$ de Kruskal-Wallis, não-paramétrico, para a análise estatística.

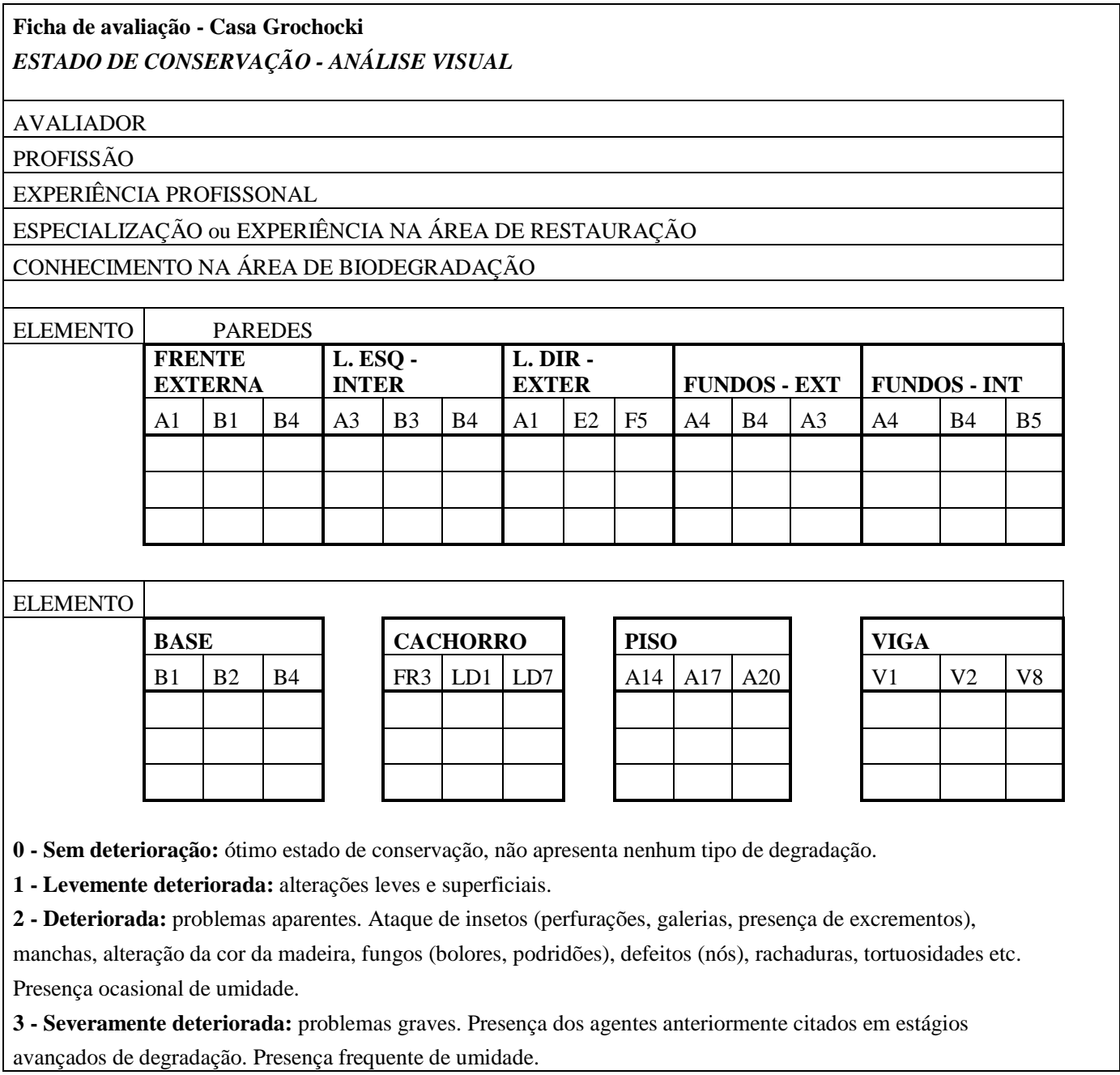

Figura 2. Ficha de avaliação.

Figure 2. Evaluation card.

\section{RESULTADOS E DISCUSSÃO}

O objeto da pesquisa histórica - a casa Grochocki - data de 1910 e conserva aspectos originais de sua constituição. Foi construída com troncos de pinho falquejados e encaixados nas extremidades (Figura 3). A casa é composta por um corpo principal (Figura 4) e acréscimo lateral de alvenaria.

Os aspectos macroscópicos, como a coloração de tom rosado mais distinta, principalmente observada em madeiras antigas, as camadas de crescimento evidentes, com o lenho inicial mais claro e o tardio mais escuro, são fatores que caracterizam a família das gimnospermas, além da presença de nós verticilados que comprovam pertencer a madeira empregada à espécie Araucaria angustifolia.

O estado geral de conservação da edificação era razoável. Em geral, as degradações ocorreram de forma pontual. As deteriorações intensas caracterizam-se por locais de elevada umidade, provocadas por infiltrações e pela ação dos demais agentes climáticos (degradação física), como ocorre na base da 
edificação, nas extremidades das peças de cobertura e nos encontros de telhados. Nesses locais, associam-se, ainda, a deterioração por fungos e insetos (degradação biológica), que por vezes levam a lesões do material (degradação mecânica).

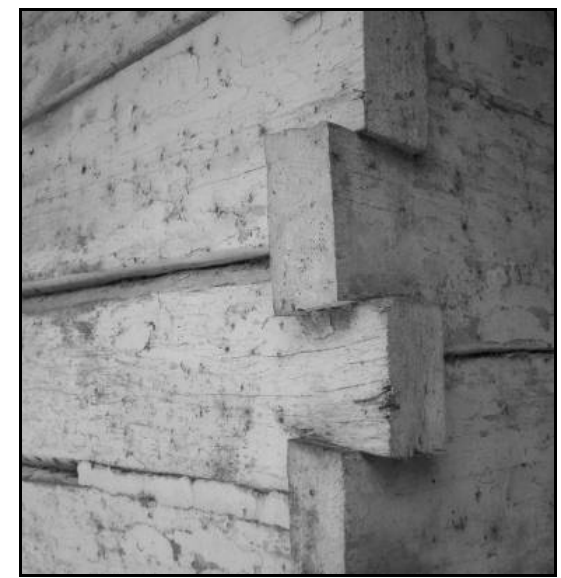

Figura 3. Detalhe da parede da casa de troncos.

Figure 3. Detail of the wall of the logs home.

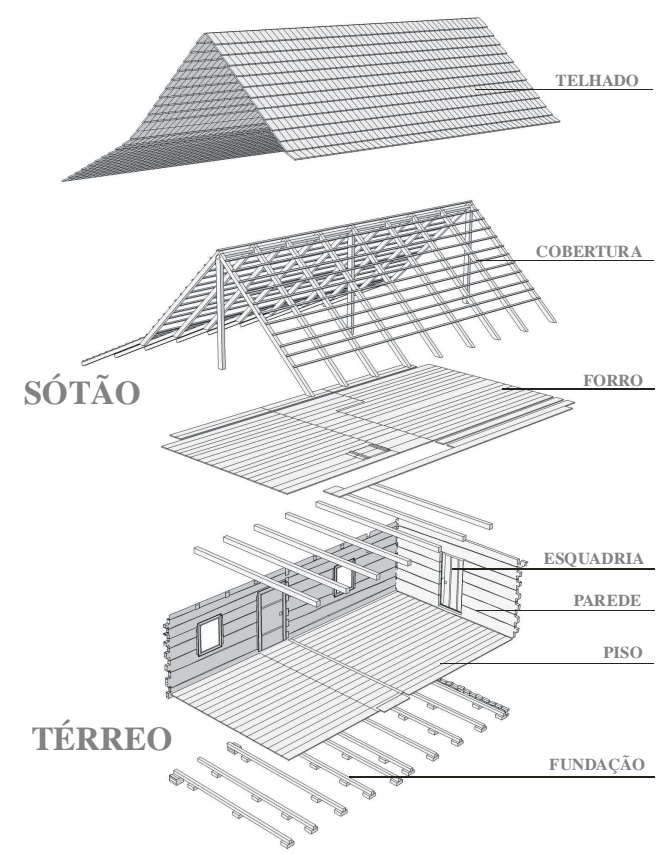

Fonte: Silva et al. (2007).

Figura 4. Elementos da arquitetura de madeira.

Figure 4. Elements of wooden architecture.

Por meio do suporte gráfico, a relação dessas interferências e patologias pode ser observada no mapeamento mostrado na figura 5 .

Com a avaliação do stress wave timer, obtiveram-se resultados positivos, pois o pequeno porte do equipamento permitiu o acesso a todos os elementos da edificação, inclusive às peças da cobertura. A leitura dos dados foi imediata, o que permitiu a obtenção de algumas informações já no local. Os cálculos realizados posteriormente auxiliaram na comparação das diferentes peças e seções, 
tábuas do piso, caibros, troncos etc. A interpretação dos dados pode configurar alerta das degradações existentes, principalmente nas áreas não visíveis, além de proporcionar mais certeza quanto ao diagnóstico. Não deve, no entanto, ser a única metodologia aplicada e regra geral a substituições, pois as intervenções na atualidade orientam à remoção somente das áreas lesionadas, além de emprego de outras técnicas modernas de reconstituição, exigindo o estudo para cada caso e um projeto de restauração específico. É uma metodologia apropriada aos bens do patrimônio histórico, o equipamento é de fácil manuseio e não causa danos ao material.

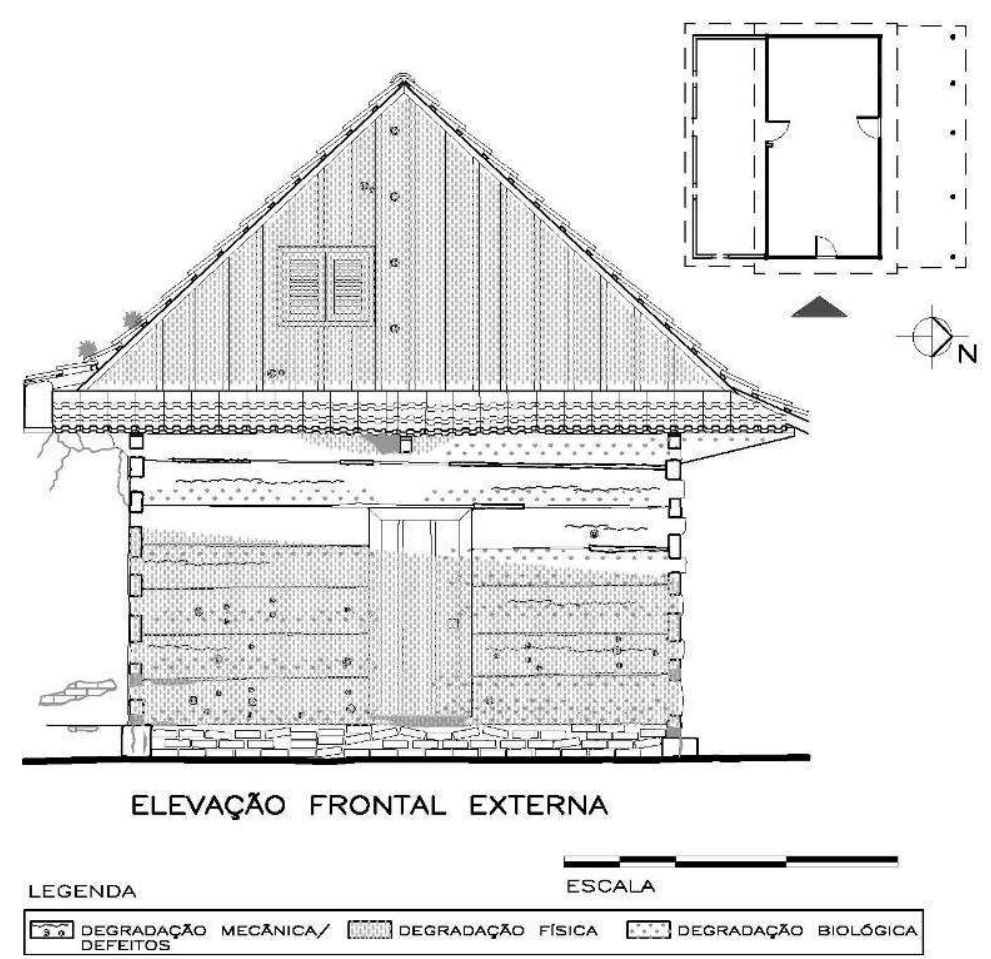

Figura 5. Mapeamento da degradação da madeira.

Figure 5. Mapping of wood decay.

Independentemente do grupo, todos os avaliadores conseguiram utilizar a ficha de avaliação. As notas obtidas, no entanto, representaram valores heterogêneos. Isso se deve ao fato de os avaliadores serem de diferentes áreas de conhecimento. Isso pode ser também comprovado na Tabela 2, com o teste H de Kruskal-Wallis (SPIEGEL, 1994), que demonstra a diferença de valores entre todos os avaliadores.

Tabela 2. Teste com todos os avaliadores.

Table 2. Test with all the evaluators.

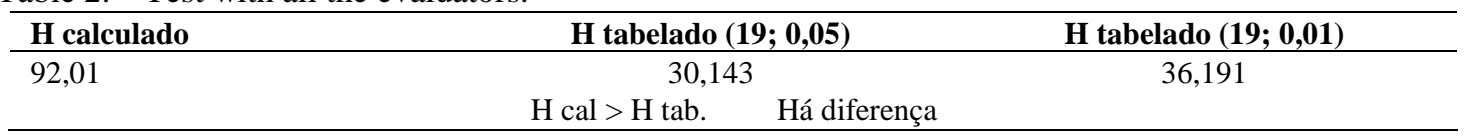

Já os testes de comparação entre a testemunha e os grupos demonstraram que não há diferença de valores com o grupo A (Tabela 3), com o grupo B (Tabela 4) ou com grupo E (Tabela 6), tendo sido percebida diferença na comparação com o grupo C (Tabela 5). 
Tabela 3. Teste de comparação do grupo testemunha com o grupo A.

Table 3. Comparison test of the witness group with group A.

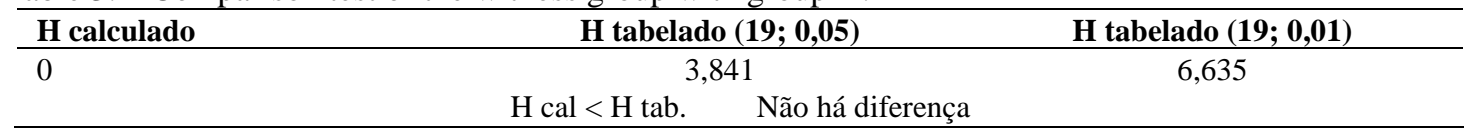

Tabela 4. Teste de comparação do grupo testemunha com o grupo B.

Table 4. Comparison test of the witness group with group B.

\begin{tabular}{lcc}
\hline H calculado & H tabelado $(\mathbf{1 9} ; \mathbf{0 , 0 5})$ & H tabelado $(\mathbf{1 9 ;} ; \mathbf{0 , 0 1})$ \\
\hline $\mathbf{1 , 9 9}$ & $\mathrm{H}$ cal $<\mathrm{H}$ tab. $\quad$ Não há diferença & 6,635 \\
& \multicolumn{2}{c}{} \\
\hline
\end{tabular}

Tabela 5. Teste de comparação do grupo testemunha com o grupo C.

Table 5. Comparison test of the witness group with group C.

\begin{tabular}{lcc}
\hline H calculado & H tabelado $(\mathbf{1 9 ;} \mathbf{0 , 0 5})$ & H tabelado $(\mathbf{1 9 ;} \mathbf{0 , 0 1})$ \\
\hline $\mathbf{6 , 7 1}$ & 3,841 & 6,635 \\
& $\mathrm{H}$ cal $>\mathrm{H}$ tab. $\quad$ Há diferença & \\
\hline
\end{tabular}

Tabela 6. Teste de comparação do grupo testemunha com o grupo E.

Table 6. Comparison test of the witness group with group E.

\begin{tabular}{lcc}
\hline H calculado & H tabelado $(\mathbf{1 9} ; \mathbf{0 , 0 5})$ & H tabelado $(\mathbf{1 9 ;} \mathbf{0 , 0 1})$ \\
\hline $\mathbf{0 , 3 8}$ & 3,841 & 6,635 \\
& $\mathrm{H} \mathrm{cal}<\mathrm{H}$ tab. $\quad$ Não há diferença & \\
\hline
\end{tabular}

Através dos resultados obtidos, constatou-se que os grupos A, B e E, formado por profissionais com experiência em restauração e biodeterioração da madeira (grupo A), especialistas em tecnologia da madeira, sem o restauro (grupo B), estudantes com formação em tecnologia da madeira ou biologia (grupo E), seriam eficientes avaliadores do estado de conservação de bens imóveis. Estes grupos seriam os mais indicados, capazes por seu conhecimento de realizar diagnósticos de edificações históricas de madeira. Observou-se, por meio dessa ficha, que os avaliadores sem formação ou experiência (Grupo F) não são recomendados a esse tipo de atividade. Observou-se também que os profissionais da arquitetura (Grupo C e D), mesmo conhecedores das teorias do patrimônio e do restauro, necessitam do aperfeiçoamento técnico na área da madeira e das patologias do lenho.

O problema das análises realizadas pelos grupos $\mathrm{C}, \mathrm{D}$ e $\mathrm{F}$ quando não há a detecção das degradações e das avarias em obras podem implicar em graves perdas dos bens históricos, ou ainda incorrer em riscos na utilização das edificações. Já as avaliações dilatadas levam a restaurações imprecisas e a substituições desnecessárias.

\section{CONCLUSÕES}

De acordo com os resultados obtidos, as seguintes conclusões podem ser apresentadas:

- A restauração possui caráter multidisciplinar e envolve profissionais de diversas áreas, confirmando os preceitos firmados no passado pelas cartas patrimoniais e pela teoria e história da restauração.

- A análise do objeto constitui etapa relevante. Foi não somente uma operação técnica necessária às demais análises, mas uma forma de obter uma leitura mais ampla, voltada à consciência cultural da obra. A arquitetura vernácula, o sítio e a história, em fim a obra construída é fonte de identidade e de valores sociais. Caracteriza o importante acervo da produção cultural do imigrante polonês na região de Curitiba.

- O levantamento do estado de conservação e o mapeamento aplicados neste estudo confirmaram sua importância como instrumentos vitais à preservação de edificações históricas de madeira.

- A metodologia não-destrutiva, com o equipamento stress wave timer, é eficaz e apropriada aos bens do patrimônio histórico. Não deve ser empregada, no entanto, sem as etapas de análise visual e do estado de conservação. 
- A ficha de avaliação é um recurso de fácil aplicação, sem distinção de classes de avaliadores.

- Os melhores avaliadores caracterizaram-se pelos profissionais que tiveram seus conhecimentos aprofundados em aspectos tecnológicos e patológicos da madeira.

- Não se pode afirmar que os grupos qualificados a partir dos testes seriam os profissionais indicados para decisões relativas a técnicas de intervenção nas obras do patrimônio histórico. Os projetos de restauração devem envolver equipes multidisciplinares, e é nesse sentido que a ficha de avaliação e a metodologia pretendem contribuir.

\section{REFERÊNCIAS}

AROSIO, P. (org.). (2003). Il Restauro. Manual do curso de restauro. Curitiba: Centro de Cultura Italiana. v. 1, v. 3, v. 4. (Paginação irregular).

AUGELLI, F. La diagnosi delle opere e delle strutture lignee. Le ispezioni. Xilema. Milano: Il prato, 2006. $165 \mathrm{p}$.

GORNIAK, E.; MATOS, J. L. M. Métodos não-destrutivos para determinação e avaliação de propriedades da madeira. In: Anais do Seminário de eucalipto: avanços científicos e tecnológicos. Lavras: Universidade Federal de Lavras, 2002. p. 76-84.

INSTITUTO DO PATRIMÔNIO HISTÓRICO E ARTÍSTICO NACIONAL. Cartas Patrimoniais. Brasília: IPHAN, 1995. 344 p. (Cadernos de documentos nº 3).

LIOTTA, G. Gli insetti e i danni del legno: Problemi di restauro. 3. ed. Firenze: Nardini, 1998. 150 p.

SILVA, J. B. (coord.). As casas de troncos - o legado da cultura polonesa: Colônia Murici. São José dos Pinhais: Universidade Federal do Paraná, Secretaria da Indústria, Comércio e Turismo de São José dos Pinhais, 2007. Painéis culturais em 3 pranchas, color., 90 x $100 \mathrm{~cm}$.

SPIEGEL, M. R. Estatística. Tradução e revisão técnica: Pedro Consentino, 3. ed. São Paulo: P. Education do Brasil, 1994. 643 p.

TAMPONE, G. Il restauro delle strutture di legno. Milano: HOEPLI, 1996. 401 p. 